\title{
Protracted cholera outbreak in the Central Region, Ghana, 2016
}

\section{Gyesi R. Issahaku ${ }^{1,4}$, Franklin Asiedu-Bekoe ${ }^{2}$, Samuel Kwashie ${ }^{2}$ Francis Broni $^{1,2}$ Paul Boateng $^{1,2}$, Holy Alomatu ${ }^{1}$, Ekua E. Houphouet ${ }^{3}$, Afua A. Asante ${ }^{2,3}$, Donne K. Ameme ${ }^{1}$, Ernest Kenu ${ }^{1}$}

Ghana Med J 2020; 54(2)supplement: 45-52 DOI: http://dx.doi.org/10.4314/gmj.v54i2s.8

${ }^{1}$ Ghana Field Epidemiology and Laboratory Training Programme, Department of Epidemiology and Disease Control, University of Ghana School of Public Health, Legon, Accra, Ghana.

${ }^{2}$ Disease Surveillance Department, Ghana Health Service, Accra, Ghana

${ }^{3}$ Faculty of Public Health, Ghana College of Physicians and Surgeons, Accra, Ghana

${ }^{4}$ Tamale Teaching Hospital, Tamale, Ghana.

Corresponding author: Donne Kofi Ameme

E-mail: amemedonne@yahoo.com

Conflict of interest: None declared

\section{SUMMARY}

Objective: On 24th October 2016, the Central Regional Health Directorate received report of a suspected cholera outbreak in the Cape Coast Metropolis (CCM). We investigated to confirm the diagnosis, identify risk factors and implement control measures.

Design: We used a descriptive study followed by 1:2 unmatched case-control study.

Data source: We reviewed medical records, conducted active case search and contact tracing, interviewed case-patients and their contacts and conducted environmental assessment. Case-patients' stool samples were tested with point of care test kits (SD Bioline Cholera Ag 01/0139) and sent to the Cape Coast Teaching Hospital Laboratory for confirmation.

Main outcomes: Cause of outbreak, risk factors associated with spread of outbreak

Results: Vibrio cholerae serotype Ogawa caused the outbreak. There was no mortality. Of 704 case-patients, $371(52.7 \%)$ were males and 55(7.8\%) were aged under-five years. The median age was 23 years (interquartile range: 16-32 years). About a third 248(35.2\%) of the case patients were aged 15-24 years. The University of Cape Coast subdistrict was the epicenter with $341(48.44 \%$ ) cases. Compared to controls, cholera case-patients were more likely to have visited Cholera Treatment Centers (CTC) $(\mathrm{aOR}=12.1,95 \% \mathrm{CI}$ : 1.5-101.3), drank pipe-borne water (aOR=11.7, 95\%CI: 3.3-41.8), or drank street-vended sachet water (aOR=11.0, 95\%CI: 3.7-32.9). Open defecation and broken sewage pipes were observed in the epicenter.

Conclusion: Vibrio cholerae serotype Ogawa caused the CCM cholera outbreak mostly affecting the youth. Visiting CTC was a major risk factor. Prompt case-management, contact tracing, health education, restricting access to CTC and implementing water sanitation and hygiene activities helped in the control.

Keywords: Cholera outbreak, Vibrio cholerae serotype Ogawa, Cholera treatment center, Water sanitation and hygiene, Cape Coast Metropolis

Funding: This work was supported by Ghana Field Epidemiology and Laboratory Training Program (GFELTP), University of Ghana

\section{INTRODUCTION}

Cholera is an acute infectious illness which presents with profuse watery diarrhoea resulting in hypovolemic shock and acidosis. It is caused by the ingestion of toxigenic Vibrio cholera serogroup O1 or O139. ${ }^{1}$ It has a short incubation period of a few hours to about five days. It is transmitted feco-orally through the ingestion of contaminated water or food and always results in death if not promptly and appropriately treated with fluids. ${ }^{2}$

Cholera remains a major public health challenge in many parts of the developing world. There are an estimated 3-
5 million cholera cases globally with over 100,000 cholera related deaths annually. ${ }^{3}$ In sub-Saharan Africa where access to safe drinking water and proper sanitation are major challenges, the burden of cholera is quite high. ${ }^{4}$

According to the World Health Organization, between 1970 and 2011, African countries reported 3,221,050 suspected cholera cases representing $46 \%$ of all cases reported globally. With the exception of the Haitian epi- 


\section{Original Article}

demic, sub-Saharan Africa accounted for $86 \%$ of reported cases and $99 \%$ of all cholera-related deaths worldwide in $2011 .{ }^{5}$

Cholera is now thought to be endemic in many parts of Ghana. In Ghana, poor sanitary conditions and the lack of access to portable drinking water in urban and periurban slums in particular, have contributed to the endemicity of cholera. Another contributory factor is the creation of man-made hazards through poor regulation of settlements in urban areas resulting in flooding because of poor drainage systems and the inadequate provision of waste management services. Since 1970, the country has recorded outbreaks of the disease. ${ }^{6}$ In 2014, Ghana had its biggest outbreak in 30 years recording the highest number of cases and the loss of several lives. During this period, Ghana recorded over 14,000 cholera cases with 127 deaths ${ }^{7}$. These cases were recorded in 78 out of the 216 districts situated in eight out of the 10 regions of the country.

On $24^{\text {th }}$ of October 2016, the central regional health directorate (CRHD) received report of suspected cholera outbreak in the Cape Coast Metropolis (CCM). The Disease Surveillance Department (DSD) of the Ghana Health Service (GHS) constituted a joint regional and national rapid response team with reinforcement from residents of the Ghana Field Epidemiology and Laboratory Training Programme and the Ghana College of Physicians and Surgeons to investigate the outbreak. The objectives of the investigation were to characterize the CCM outbreak, determine the associated risk factors and institute appropriate control measures.

\section{METHODS}

\section{Outbreak Setting}

The outbreak occurred in the Central Regional capital of Cape Coast. It is Ghana's colonial capital and a hub for tourist activities because of its historic slave sites, castles, and forts. The Cape Coast metropolis is bordered by the Gulf of Guinea to the south, Komenda/Edina/Eguafo/ Abirem municipality to the west, Abura/Asebu/ Kwamankese district to the east and the Twifo/Heman/ Lower Denkyira district to the north. It has an estimated population of 160,849 inhabitants according to the 2010 population and housing census and occupies a land area of $9,826 \mathrm{~km} \cdot{ }^{2,8}$ It's a peri-urban settlement with several health centers, clinics, and both private and public hospitals. The metropolis has one teaching hospital.

\section{Study Design}

Both descriptive and analytic study designs were employed to investigate the outbreak. Cases were described by person, place, and time. The analytic design was a 1:2 un-matched community-based case control study. The participants of the study included all residents of CCM confirmed with cholera during the outbreak and randomly selected neighborhood controls where the cases lived. The investigations were done between $29^{\text {th }}$ October 2016 and $4^{\text {th }}$ January 2017.

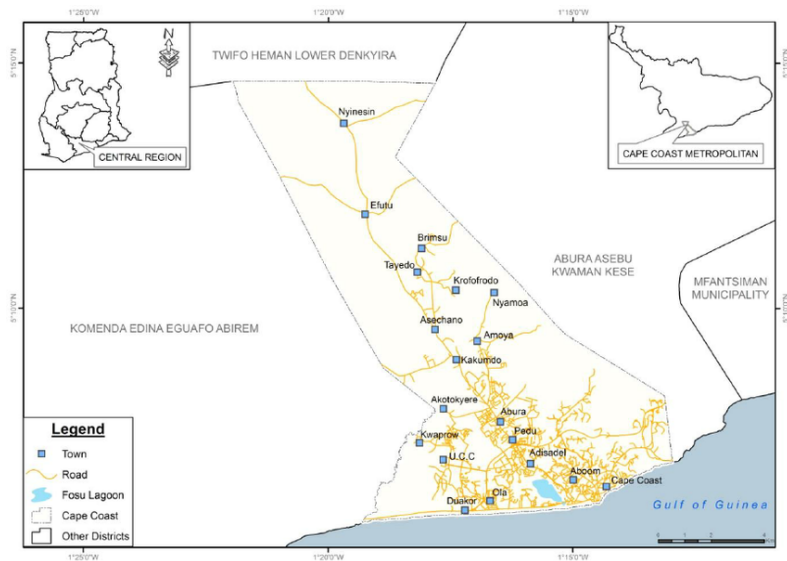

Figure 1 Map of the Cape Coast Metropolis

\section{Data collection}

Descriptive Study: We engaged and interviewed the Metropolitan Director of Health Services, Metropolitan Disease Control Officer (MDCO), the Public Health Nurse, the Hospital Management Teams, Clinicians, Laboratory staff and the Environmental Health Officer to obtain first-hand information on the outbreak and the initial data on those affected. We reviewed surveillance data, medical records, and the initial line-list generated by the MDCO.

Based on the initial information gathered, a cholera casepatient was defined as any person in the Cape Coast Metropolis with acute watery diarrhoea, with or without vomiting between $12^{\text {th }}$ October 2016 and $4^{\text {th }}$ January 2017 or epidemiologically linked to a person with vibrio cholera isolated in Cape Coast within the same period. We then reviewed medical records from all health facilities in the metropolis to identify persons who met the case definition.

Data abstracted included age, sex, occupation, place of residence, date of onset, date of presentation at health facility, signs and symptoms and outcome. We conducted active case search in the communities and interviewed case patients, their contacts and community members using an abridged semi-structured questionnaire that focused on socio-demographic and basic case history data. Case Control Study

An unmatched case control study was conducted in the metropolis using the entire population of CCM as study population. Using the 'StatCalc' utility feature of Epi 


\section{Original Article}

Info statistical software for calculating sample size when comparing proportions, we used a confidence level of $95 \%$, power of $80 \%$, expected exposure frequency (drinking sachet water) in controls of $30 \%$, expected exposure frequency in cases of $55 \%$, case to control ratio of $1: 2$ and an odds ratio of 3 (for a risk factor on which intervention would have a significant impact). A minimum sample size of 45 cases and 90 controls was obtained and interviewed.

\section{Selection of Cases and Controls}

Case patients were selected from Cholera Treatment Centres in the health facilities. All the facilities within the Cape Coast Metropolis that reported cases were included. These were Cape Coast Teaching Hospital (CCTH) with $323(50 \%)$ of the hospitalized cases-patients, University Hospital 141(20.0\%) cases-patients, Ewim Polyclinic 94(13.4\%) cases-patients, Cape Coast Metropolitan Hospital (CCMH) 63(8.9\%) cases-patients and Baiden Ghartey Hospital 47(6.7\%) cases-patients. A total of 23 cases were selected proportionate to number of cases per treatment centre. In each centre, the cases were randomly selected. Thus, CCTH - one (1) case, University Hospital nine (9) cases, Ewim Polyclinic six (6) cases, CCMH four (4) cases and Baiden Ghartey Hospital three (3) cases. Whiles some of the cases were interviewed in the treatment centres, others were interviewed in their homes. A control was defined as any person living in Cape Coast metropolis from $12^{\text {th }}$ October from $4^{\text {th }}$ January 2017 without diarrhoea or vomiting and coming from same community and neighborhood of a case. We selected two controls for each case and interviewed them on the same day on which the case was interviewed. The first control was randomly selected from the same household as the case. The second control was selected by spinning a bottle in the center of the house of a case. We selected the immediate house to the direction of the bottle and randomly selected the second control from that house. Random selection of controls was done by numbering all persons met in a household on pieces of paper, mixed them and selected one. We visited as many houses as required to recruit 90 controls for all the 45 cases.

Field workers obtained informed consent before interviewing both cases and controls. In the case of a decline to participate, the fieldworkers repeated the bottle-spinning procedure to select another household. Individuals were excluded from being controls if they reported suffering from watery diarrhoea since $12^{\text {th }}$ October 2017.

A standardized questionnaire, written in English, was administered to both cases and controls either in their native language or English by bilingual trained field workers recruited from the Cape Coast Metropolitan Health Direc- torate. The questionnaire collected basic demographic information and contained questions pertaining to sources of drinking water, main source of food (whether home prepared or vended foods), hand-washing practices, type of toilet facility used, household waste disposal practices, and taking care or visiting someone at Cholera Treatment Center from October 12, 2016 to the interview date. If a case or control was a child, the questionnaire was administered to their parents or care givers.

Laboratory Investigations: For each case-patient, we collected stool samples and a rectal swap and sent to the Cape Coast Teaching Hospital laboratory for microbiologic assessment. The microbiological investigations were done in the microbiology laboratory of the Cape Coast Teaching Hospital.

We transported stool samples on Cary-Blair transport media to the laboratory for culture and antibiotic susceptibility testing. Samples were plated on Thiosulfate-Citrate Bile Salts (TCBS) agar. We evaluated growth colonies using standard biochemical reactions. Positive vibrio isolates were serogrouped and serotyped using agglutination tests with commercial anti-sera.

\section{Environmental Assessment}

We conducted environmental assessment of affected communities, focusing on their sources of water supply and sanitation practices. We observed activities along the sea shore and river banks, community drainage system, the sewage lines, general sanitation along water bodies.

\section{Data Management and Analysis}

Data was entered, cleaned, and analysed using Epi Info version 7. A descriptive analysis of the outbreak data by person, place and time was performed. Univariate analysis was done by expressing categorical variables as frequencies and relative frequencies. Continuous variable (age) was expressed as median and Interquartile Range. We calculated attack rates and drew an epidemic curve to characterize the outbreak.

For analysis of the case-control data, we dichotomized qualitative exposure-variables and compared them among case patients and controls in bivariate analysis. We used Chi-Square test at 95\% confidence level to identify potential risk factors. We included all significant factors in a logistic regression model at $95 \%$ confidence level to identify the risk factors associated with cholera case status.

An exposure was considered a risk factor if the odds of association with cholera-case status at $95 \%$ confidence interval was statistically significant ( $p$-value $<0.05$ ).

\section{Ethical Issues}


This investigation was carried out in response to an outbreak which is regarded as a public health emergency. Hence, approval to investigate the outbreak was given by National Disease Surveillance Department, the Regional Health Directorate and the District Health Directorate Central Regional Health Directorate and the Cape Coast Metropolitan Health Directorate gave approval for the investigation. Informed consent was obtained from the participants before the interviews. The confidentiality of participants was protected through the use of codes. Preliminary report of the outbreak was discussed with the Surveillance Unit of the Ghana Health Service, Regional and Metropolitan Health Directorates, the Metropolitan Assembly and the chiefs and elders of the affected communities.

\section{RESULTS}

Descriptive Epidemiology

The cholera outbreak in CCM started during epidemiological week 38 of 2016 ( $17^{\text {th }}$ October $)$. The primary case was a 26-year-old fisherman, who returned from a 9-day fishing expedition on the $14^{\text {th }}$ October 2016. On the same day, he started experiencing diarrhoea and vomiting but did not report to any hospital. Instead, he self-medicated with antibiotics obtained from a chemical store.

The index case was a 22 years old female trader at the Kotokraba market who sells fish and happened to be the wife of the primary case. She took care of the primary case and started experiencing symptoms on the $17^{\text {th }}$ of October 2016. She reported to the Ewim polyclinic on the $21^{\text {st }}$ of October 2016. A laboratory investigation with SD Bioline Cholera Ag 01/0139 rapid diagnostic test (RDT Germany) on her stool sample was positive for cholera.

A total of 704 case-patients were recorded. However, there were no deaths. The overall attack rate was $0.41 \%$ (704/16,894). The sex-specific attack rates were $0.44 \%$ for males and $0.38 \%$ for females. Of the four sub metros in CCM, the University of Cape Coast (UCC) sub metro was the epi center of the outbreak recording 341(48.44\%) case patients. The outbreak involved people from over 40 communities in the Cape Coast municipality and its surrounding districts. The five most affected communities were Amamoma 63(8.95\%), Abura 51(7.24\%), Pedu $36(5.13 \%)$, Apewosika 35(4.97\%) and Essuekyir $22(3.31 \%)$. There was one epidemiologically linked case from Shama in the Western Region, and another from Fumesua in the Ashanti Region.

From the epi-curve in Figure 2, the onset of diarrhoea and vomiting in the metropolis was on the $17^{\text {th }}$ of October 2016. The epi-curve had 3 notable peaks $\left(27^{\text {th }}\right.$ of October, $10^{\text {th }} / 11^{\text {th }}$ November and $16^{\text {th }} / 17^{\text {th }}$ November $)$. The outbreak was protracted in nature lasting a period of 12 weeks. The outbreak had a propagated pattern of spread with intermittent abatements.

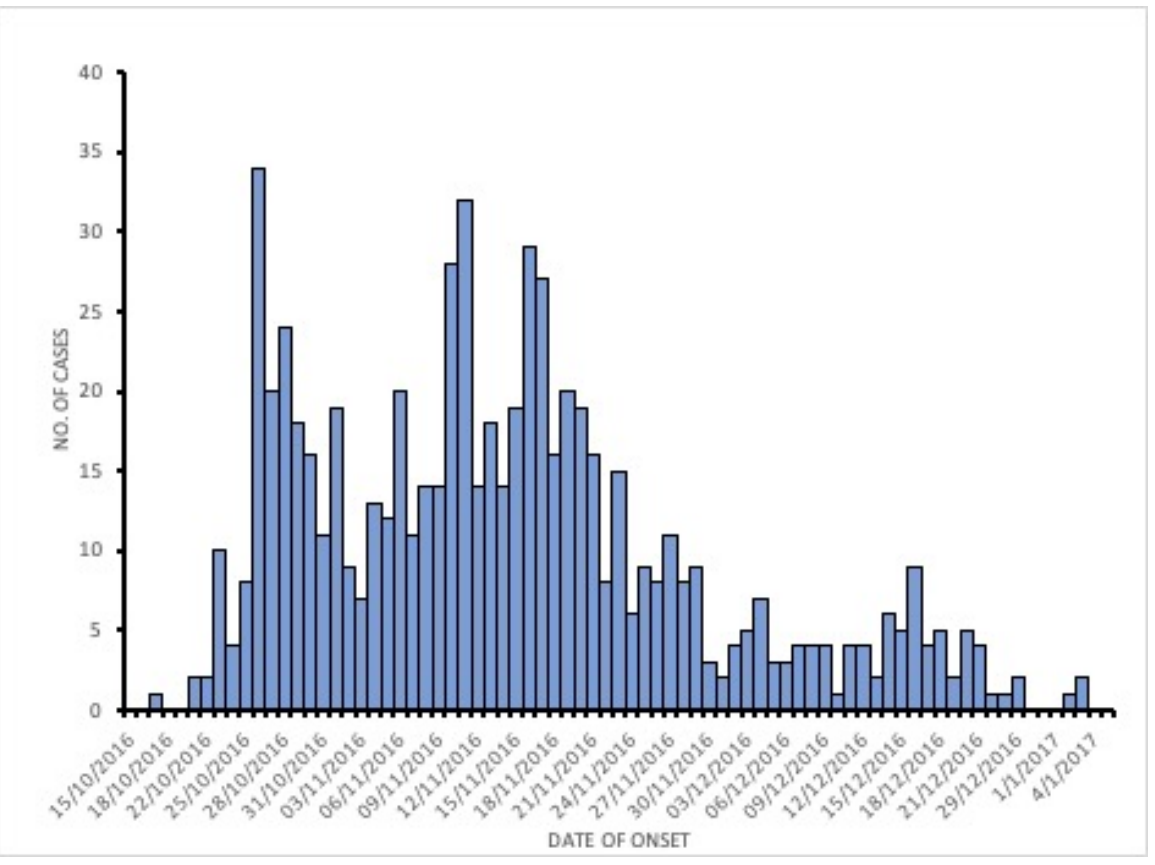

Figure 2 Distribution of cholera cases by date of onset, Cape Coast October 2016 


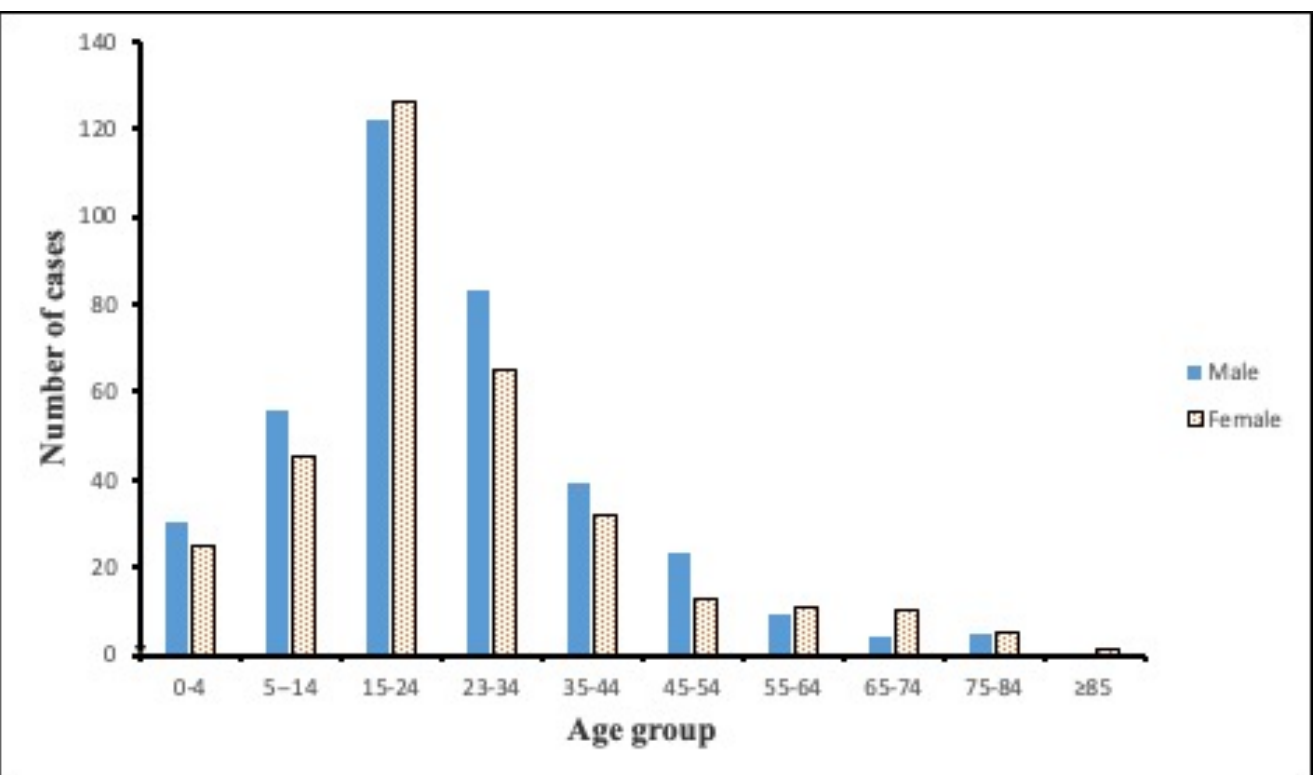

Figure 3 Age and Sex distribution of cholera cases, Cape Coast, 2016

The affected ages ranged from 7 months to 85 years with a median age 23 years (IQR $16-32$ years). Males formed $371(52.7 \%)$ of the cases. Fifty-five (7.8\%) case patients were aged under five years. Age group 15-24 had most case-patients $248(35.2 \%)$. The least affected age group was 85 years and above recording $1(0.1 \%)$, Figure 3 .

\section{Analytical Epidemiology}

\section{Characteristics of Cases and Controls}

We recruited 135 participants for the case control study. Of these, 45 were cases and 90 were controls as per our 1:2 case to control ratio. The median age of cases was 23 years (IQR 11-39) and that of controls was 32 years (IQR 23.0 - 48.8). The common symptoms among cholera cases patients were diarrhoea $45(100 \%)$, vomiting 35 (77.8\%), and abdominal cramps $30(66.7 \%)$.

Table 1 .

Table 1 Frequency of clinical signs and symptoms for cholera case patients, Cape Coast metropolis, 2016

\begin{tabular}{|l|l|}
\hline Signs and Symptoms & No. of cases $(\%)$ \\
\hline Diarrhoea & $45(100)$ \\
\hline Vomiting & $35(77.8)$ \\
\hline Abdominal Cramps & $30(66.7)$ \\
\hline Headache & $17(37.8)$ \\
\hline Chills & $9(20.0)$ \\
\hline Nausea & $7(15.6)$ \\
\hline Fever & $12(26.7)$ \\
\hline
\end{tabular}

\section{Risk factors for the outbreak}

In a multiple logistic regression analysis, the study found that, persons who visited the Cholera Treatment Centre had over twelve times higher odds of becoming case-patient compared to those who did not. [(aOR) 12.13 (CI): 1.45 -101.32] (Table 2).
Similarly, drinking either pipe-borne water [(aOR) 11.74 (CI): $3.29-41.81]$ or sachet water [(aOR) 11.01 (CI): 3.68 - 32.90], were each significantly associated with over eleven times increased odds of developing cholera among cases compared to controls (Table 2).

For all cases and controls who washed their hands before eating, the odds of falling ill of cholera was reduced by $10 \%$ in controls compared to cases $(\mathrm{aOR}=0.9 ; 95 \% \mathrm{CI}$ : 0.10-0.46).

Table 2 Risk factors for cholera, Cape Coast metropolis, 2016

\begin{tabular}{|c|c|c|c|c|c|}
\hline Risk Factor & Cases & Controls & AOR & $95 \%$ CI & p-Value \\
\hline $\begin{array}{l}\text { Visit to the } \\
\text { CTC }\end{array}$ & $44 / 45$ & $69 / 90$ & 12.13 & $\begin{array}{l}1.45 \\
101.32\end{array}$ & 0.020 \\
\hline $\begin{array}{l}\text { Drinking } \\
\text { Pipe-borne } \\
\text { Water }\end{array}$ & $37 / 45$ & $31 / 88$ & 11.74 & $3.29-41.81$ & $<0.001$ \\
\hline $\begin{array}{l}\text { Drinking } \\
\text { Sachet Wa- } \\
\text { ter }\end{array}$ & $29 / 45$ & $32 / 90$ & 11.01 & $3.68-32.90$ & $<0.001$ \\
\hline $\begin{array}{l}\text { Eating } \\
\text { Street } \\
\text { Vended } \\
\text { Food }\end{array}$ & $20 / 45$ & $22 / 90$ & 3.41 & $1.33-8.79$ & 0.011 \\
\hline $\begin{array}{l}\text { Wash hands } \\
\text { before eat- } \\
\text { ing }\end{array}$ & $17 / 45$ & $41 / 90$ & 0.90 & $0.10-1.46$ & 0.826 \\
\hline
\end{tabular}

\section{Case management}

Oral Rehydration Salts, intravenous fluids and electrolytes, were administered in a timely manner and in adequate volumes. 


\section{Original Article}

This intervention averted mortalities in cholera case patients. In addition to rehydration, antibiotic treatment was given for all hospitalized patients. A single dose of doxycycline $(300 \mathrm{mg})$ was the first-line treatment for adults, and a single dose of azithromycin $(20 \mathrm{mg} / \mathrm{kg})$ was the first-line treatment for children.

\section{Contact Tracing}

The outbreak response team contact listed and traced 1,542 contacts. These contacts were given education on the immediate signs and symptoms of cholera. They were monitored on a daily basis through home visiting by community health nurses and community volunteers. A total of 33 contacts eventually came down with cholera and were duly managed.

\section{Environmental Assessment}

In general, there was poor environmental sanitation in all the affected communities. We observed open defecation, broken sewage systems, crude dumping of refuse in most affected communities. Additionally, we observed pollution of water sources and inadequate and unsafe water supply in the epicenter. Pipelines were observed to have been laid through open drainage systems risking the possibility of contamination in the case of a breakage.

\section{Laboratory findings}

Of the 331 suspected cases investigated by stool culture, $123(37.2 \%)$ were confirmed culture positive for Vibrio cholera. All these culture positive samples showed positive agglutination tests for serotype Ogawa subtype. Majority, $45.5 \%$ (56/123) of the confirmed cases, were from the UCC sub metro, the epicenter of the outbreak.

\section{DISCUSSION}

Cholera remains a public health problem in many coastal towns in Ghana which includes the Cape Coast Metropolis. In spite of the improved understanding of the epidemiology and public health aspects of cholera, it continues to be a major public health challenge among many poor and vulnerable communities. This situation is attributable to developmental challenges that has to do with reduced access to portable drinking water and improper sanitary conditions.

Our study revealed that, the cholera outbreak affected all age groups in the Cape Coast Metropolis. The outbreak affected more males in the metropolis compared to females. The majority of male case patients observed in this study may be a reflection of the higher tendency of males to patronize street vended foods. This finding is very much in keeping with similar studies from Ghana and other countries where males were mostly the more affected sub-population. ${ }^{9-12}$
This is however at variance with the findings of a Ugandan study where Bwire and colleagues found that females were more affected. ${ }^{13}$ The most affected age group was $15-24$ years accounting for about $35 \%$ of the casepatients. Other studies have documented youthful populations as the most affected age group. ${ }^{10,14,15}$ In direct contrast to this, other studies have reported children and elderly people mostly affected by cholera. ${ }^{16,17}$

About $37.2 \%$ (123/331) of the collected stool samples and rectal swabs from various health facilities in the metropolis were confirmed positive for cholera. All the isolates were biochemically identified as $V$. cholerae and serologically confirmed as O1 Ogawa. Vibrio cholerae serotype Ogawa has been reported by previous studies to be the common cause of outbreaks especially in urban areas. ${ }^{3,9,10}$

Visiting the CTC, drinking pipe-borne water or street vended sachet water, and eating street-vended food significantly increased the odds of acquiring cholera. The absence of basic infection prevention measures such as the handwashing and the use of PEP's in the CTC'S probably explains the increased odds exposure on visiting the CTC. The observed pipelines that passed through opened drainage system opens up the risk of contamination in the damaged pipelines. A study by Dzotsi et al. on Cholera outbreak in Greater Accra in 2014 reported increased odds of 6.0 of acquiring cholera by drinking street vended sachet water and eating street vended food. ${ }^{10} \mathrm{Wa}-$ ter borne cholera outbreak has been reported in several studies worldwide. ${ }^{9,11,18,19}$ An outbreak investigation by Opare and team in the Upper East regional town of Bongo reported increased odds of getting cholera among attendees of the burial ceremony of case patients. ${ }^{20} \mathrm{In}$ our study, the risk factor analysis demonstrate that the most likely source of the infection was contaminated food or drink and the observed mode of spread was person to person contact. Both Azman and Opare reported these two factors as major contributors to the occurrence and spread of outbreaks. ${ }^{20,21}$

There were no mortalities associated with this outbreak. Contrary to this finding, a number of studies in Ghana $^{20,22,23}$ have reported various degrees of mortalities in their works. Findings from other African countries like Nigeria and Zambia have reported mortalities associated with cholera outbreaks. ${ }^{12,24,25}$ Early reporting and appropriate rehydration was the reason for the zero mortalities recorded. A previous study by Siddique in Goma has also documented early reporting to be associated with increased survival rates. ${ }^{26}$ 
Our study had a few limitations. Owing to its unmatched design, we were unable to control for confounding variables such as differences in age and socio-economic status among respondents. We were also unable to collect food/water samples from the communities for laboratory investigation. There was the possibility of recall bias given that patients and controls had to recall their exposure behaviors in responding to the questionnaire. To reduce the risk of recall bias, we selected cases-patients that had recently come down with cholera. Despite these limitations, our study provided beneficial information that informed stakeholder actions in controlling the outbreak. The study findings also provided evidence for recommendations towards the prevention of future outbreaks.

\section{Public Health Actions Taken}

As part of our WASH activities, the team distributed a total of 12,638 chlorine tablets to households. We also disinfected 9,184 latrines and open drains in communities where cases and their contacts came from. There was the distribution of materials on the prevention of diarrheal diseases to residents of the most affected communities in Amamoma, Abura, UCC, Pedu, Apewosika and Essuekyir.

\section{CONCLUSION}

A protracted cholera outbreak with a propagated pattern of spread occurred in the Cape Coast Metropolis (CCM). Vibrio cholerae serotype Ogawa caused the CCM cholera outbreak affecting mostly the youth. Visiting CTC was a major risk factor for developing the disease. Casemanagement, contact tracing, health education, restricting access to CTC and implementing Water Sanitation and Hygiene activities helped control the outbreak.

\section{REFERENCES}

1. MacGarty D, Nott DM (eds). Cholera: An Infectious Waterborne Disease In Disaster Medicine: Acase based approach. Springer -Verlag. London, 2013 pp. 263-276:

2. Nel L.H, Markotter W. New and emerging waterborne infectious diseases. Water and Health2009: 1;147.

3. Ali M, Nelson AR, Lopez AL, Sack D.A. Updated global burden of cholera in endemic countries. PLoS neglected tropical diseases 2015: 9(6), e0003832.

4. Adagbada AO, Adesida SA, Nwaokorie FO, Niemogha M-T, Coker A.O.J.P.A.M.J. Cholera epidemiology in Nigeria: an overview. Pan Afr Med J. $2012 ; 12: 59$.

5. World Health Organization. Global health observatory 2012. WHO, Geneva. 2012.
6. Ofori-Adjei D, Koram K. Of Cholera and Ebola Virus Disease in Ghana. Ghana Med J. 2014 Sep; 48(3): 120.

7. Ghana Health Service. 2014 National Annual Report. Ghana health Service, Accra. 2015

8. Ghana Statistical Service, Ghana Health Service, and ICF Macro. Ghana demographic and health survey 2008. GSS, Accra. 2010

9. Opare J, Ohuabunwo C, Afari E, Wurapa F, Sackey S, Der J, Afakye K, Odei E. Outbreak of cholera in the East Akim Municipality of Ghana following unhygienic practices by small-scale gold miners, November 2010. Ghana medical journal 2012: 46(3), 116.

10. Dzotsi E, Odoom JK, Opare JK and Davies-Teye BB. Outbreak of Cholera, Greater Accra Region, Ghana, 2014. J Sci Res Rep 2016: 9(3), 1-12.

11. Sur D, Deen JL, Manna B, Niyogi SK, Deb AK, Kanungo S, Sarkar BL, Kim DR, DanovaroHolliday MC and Holliday K. The burden of cholera in the slums of Kolkata, India: data from a prospective, community based study. Archives of disease in childhood 2005; 90(11), 1175-1181.

12. Shadrack BE, Ajumobi O, Olayinka A and Nguku P. Investigation of Cholera Outbreak in an Urban North Central Nigerian Community-The Akwanga Experience. Journal of Public Health Research 2014: 4(1), 7-12.

13. Bwire G, Malimbo M, Maskery B, Kim YE, Mogasale V, Levin AJ. The burden of cholera in Uganda. PLoS NTD 2013: 7(12), e2545.

14. Kansakar P, Baral P, Malla S, Ghimire GR. Antimicrobial susceptibilities of enteric bacterial pathogens isolated in Kathmandu, Nepal, during 2002-2004. J Infect Dev Ctries. 2011;5(3):163-8.

15. Yadav D., Tamrakar D., Baral R., Jha P., Gautam S. and Pokharel P. Outbreak of Cholera in Tilathi VDC Saptari Nepal. Kathmandu University Medical Journal 2012: 10(4), 36-39.

16. Rai K., Sherchand J.B. and Bhatta D.R. Study of enteropathogens and its predisposing factors in gastroenteritis suspected children attending Kanti Children Hospital, Kathmandu, Nepal. J Nepal Assos Med Lab Sciences 2004: 648-53

17. Harris JF, Ryan ET, Calderwood SB. Cholera. Lancet 2012: 3792466-2476.

18. Alam NH, Ashraf H. Treatment of infectious diarrhea in children. Paediatr Drugs. 2003;5:15165

19. Heymann DL(ed), Control of Communicable Diseases Manual. $19^{\text {th }}$ ed. American Public Health Association Press: Washington; 2015.

20. Opare JK, Awoonor-Williams JK, Odoom JK, Awuni B, Juliana A, Otareyoo M, Addo C, Asante DK, and Opare OS. Outbreak of Cholera in Vea- 
Gunga, Bongo District of the Upper East Region, Ghana: Impact of Traditional Medicine and the National Health Insurance Scheme. Kei Journal 2017: 5;4

21. Azman AS, Rudolph KE, Cummings DA, Lessler JJ. The incubation period of cholera: a systematic review. J Infect. 2013: 66(5), 432-438.

22. Dzotsi E, Odoom JK, Opare JK.. and Davies-Teye BBK. Outbreak of cholera, Greater Accra region Ghana J Sci Res Rep. 2016: 9(3), 1-12.

23. Noora $\mathrm{C}$, Issah $\mathrm{K}$, Kenu $\mathrm{E}$, Bachan $\mathrm{EG}$, Nuoh RD, Nyarko MN, Appiah P, Letsa T. Large cholera outbreak in Brong Ahafo Region, Ghana. BMC Res Notes 2017: 10(1), 389.

24. Oluwafemi F, Oluwole MEJ (2012) Microbiological examination of sachet water due to a cholera outbreak in Ibadan, Nigeria. OJMM 2012: 2(3), 115.

25. Mwambi P, Mufunda J, Lupili M, Bangwe K, Bwalya F, Mazaba MJ. Timely response and containment of 2016 cholera outbreak in northern Zambia. Med J of Zambia 2016: 43(2), 64-69.

26. Siddique AK. Failure of treatment centres to prevent cholera deaths in Goma. The Lancet 1995. 346: 379 